\title{
Problemas del financiamiento subvencionado en la educación inicial de Chile
}

Para citar este artículo | To cite this article Barco, B. (2021). Problemas del financiamiento subvencionado en la educación inicial de Chile. magis, Revista Internacional de Investigación en Educación, 14, 1-18. doi: 10.11144/Javeriana.m14.pfse 


\title{
Resumen
}

El costo a la educación inicial es un obstáculo para las familias desaventajadas. Los centros de la Red de Oferta Pública en Chile garantizan gratuidad para los niños de 0 a 3 años.

Examinando la institucionalidad del financiamiento a centros subvencionados con una aproximación etnográfica, se identifican mecanismos causales en la implementación de esta política. La variabilidad de la asistencia y la lógica de control económico generan prácticas entre las administraciones, centros y familias que promueven un derecho a la educación y ciudadanía en la primera infancia de consumo.

\section{Palabras clave}

Acceso a la educación; ciudadanía; derecho a la educación; educación de la primera infancia; financiación de la educación

\begin{abstract}
The cost of early childhood education is an obstacle for disadvantaged families. The centers of the Public Offering Network in Chile guarantee free admission for children from 0 to 3 years old. We examined the institutional framework that financed subsidized centers from an ethnographic approach. Causal mechanisms were identified in the implementation of this policy. For instance, the variability of assistance and the logic of economic control generate practices among administrations, centers and families which promote a right to education and citizenship in early childhood consumption.
\end{abstract}

\section{Keywords}

Access to education; citizenship; right to education; early childhood education; educational finance 
Descripción del artículo | Article description

Este artículo de investigación es parte del proyecto de tesis doctoral Acceso de las familias desaventajadas a salas cunas y niveles medios en la Red de Oferta Pública de Chile: ¿Diversificación de mercado educacional para la equidad social?, financiado por CONICYT-PCHA/Doctorado Nacional/2014-21140206.

\section{Introducción}

La educación inicial (en adelante ECE, por sus siglas en inglés [early childhood education]) es considerada una etapa significativa en el aprendizaje del individuo, pues se adquieren habilidades complejas en el plano físico, emocional, psíquico, cognitivo y social (Bedregal \& Pardo, 2004; Berlinski \& Schady, 2015; Blanco, 2005; Heymann, 2003). Investigaciones han identificado que la ECE tiene un efecto en el aprendizaje de los niños mayor que cualquier otra intervención posterior, especialmente en niños de familias desaventajadas (Cunha et al., 2006; Heckman, 2006). Estos hallazgos impulsaron a la Organización para la Cooperación y el Desarrollo Económico (OCDE) a mejorar el acceso a la ECE (Organisation for Economic Co-operation and Development, 2001), debido a que su distribución perjudica a familias desaventajadas (Collombet, 2018; Ghysels \& Van Lancker, 2011; Organisation for Economic Co-operation and Development, 2006; 2017a; 2017b; Van Lancker, 2013).

En los últimos treinta años en Chile, los distintos Gobiernos han impulsado políticas para favorecer el acceso a la ECE, ampliando la cobertura, seleccionando con discriminación positiva a las familias desaventajadas y garantizando la gratuidad en las distintas provisiones de la Red de Oferta Pública para niños de 0 a 3 años (Ministerio de Educación et al., 2005). A pesar de estas políticas, la matrícula en estos cursos presenta brechas entre los distintos quintiles de ingreso por hogar. Los niños de los hogares del primer quintil (más pobres) tuvieron una matrícula del 25,5\%; mientras que los niños de los hogares del quinto quintil (más ricos), una del 42,2\% en el año 2017 (Ministerio de Desarrollo Social, 2018).

Este artículo busca contribuir a dos ejes del dossier: analizando 1) la institucionalidad del financiamiento en los centros subvencionados para familias desaventajadas en la Red de Oferta Pública (en adelante, ROP) de Chile, a partir de 2) las subjetividades de administrativos, directivos, equipo educativo y familias. A partir del realismo crítico, se utilizaron estrategias de 
contextualización y análisis de proceso para conceptualizar, que permitieron generar mecanismos causales con respecto a las prácticas con el objetivo de identificar cómo se promueve el derecho a la educación y la ciudadanía en la primera infancia.

\section{Marco conceptual}

En este apartado se desarrolla brevemente el concepto de derecho a la educación y la ciudadanía de la primera infancia, la asequibilidad o costo como barrera para acceder a la ECE y los antecedentes del contexto chileno.

\section{Derecho a la ECE y ejercicio de la ciudadanía}

de la primera infancia

El derecho a la ECE, como derecho universal para la primera infancia, ha sido promovido con mayor énfasis desde el año 2000 por organismos internacionales (Fondo de las Naciones Unidas para la Infancia, 2007; Organisation for Economic Co-operation and Development, 2001). La implementación de este derecho se relaciona con un concepto de ciudadanía vinculado a una "igualdad plena" o "universal", en un sentido homogeneizador para garantizar el acceso (Marshall, 1992).

Una forma de implementar un mayor acceso a la ECE en Estados con economías neoliberales ha sido la participación de privados en la provisión (Ball \& Vincent, 2005; Moss, 2009). Esto ha provocado un cambio desde una "ciudadanía social" hacia una "ciudadanía de consumo", vinculada a modelos de producción diferenciados y elección individual (Guerrero, 2001).

Tal como se constata en los párrafos anteriores, el concepto de ciudadanía ha tenido transformaciones. Esto ha llevado a algunos autores a considerar el concepto de ciudadanía como un espacio o "campo" de disputa política (Cockburn, 2013; Wacquant \& Bourdieu, 2005), en el que las interacciones y prácticas se encuentran situadas en determinados contextos culturales y sociales (Delanty, 2003). Por otro lado, las prácticas cotidianas en las cuales se ejerce esta ciudadanía no son aisladas y ocurren dentro de interdependencias generacionales: primera infancia, familia, educador, adultos mayores, etc. (Devine \& Cockburn, 2018). Si bien esta interdependencia generacional es beneficiosa, se basa en un poder estructural asimétrico (Devine, 2002).

Considerando la ciudadanía de la primera infancia como campo de disputa, con interdependencia generacional y situada en determinados contextos, este artículo se enmarca en el nivel micro de la implementación de políticas y estructuras que condicionan el ejercicio del derecho a la educación y la ciudadanía. 


\section{Asequibilidad en la ECE}

Revisiones de investigaciones empíricas en el contexto europeo (Lazzari \& Vandenbroeck, 2012; Vandenbroeck \& Lazzari, 2014) identifican que uno de los factores que dificultan el acceso de las familias desaventajadas a la ECE corresponde a la asequibilidad (affordability), es decir, al costo monetario (Del Boca, 2010) u otros costos simbólicos, como ser etiquetados "en necesidad" o renunciar a su privacidad (Roose \& De Bie, 2003).

Reducir o diferenciar el costo monetario a familias desaventajadas dependerá del sistema de financiamiento a la ECE en los distintos países. Aquellos donde el sistema de provisión corresponde a uno de tipo universal y gratuito o donde los costos están vinculados a los ingresos familiares tienden a ser más eficientes y con mayor potencial de equidad que en los sistemas limitados o dirigidos a las familias desaventajadas (Moss, 2009). Por otro lado, sistemas universales no gratuitos tienden a favorecer a quintiles más altos de ingreso (Ghysels \& Van Lancker, 2011), lo que fomenta un mercado privado con lucro (Dominiciano, 2011) y asigna una mayor carga financiera a las familias de bajos ingresos y un alto impuesto efectivo sobre las ganancias para las mujeres (Meyers \& Gornick, 2003). Es por ello que una mayor provisión pública y gratuita se relaciona con niveles bajos de desigualdad (Dominiciano, 2011; Van Lancker \& Ghysels, 2016).

\section{Antecedentes del contexto chileno}

En Chile, la ECE para niños entre 0 a 3 años se organiza en cursos por edad o desarrollo individual: Sala Cuna Menor - desde 85 días hasta 11 meses de edad - Sala Cuna Mayor — desde 1 año hasta 1 año y 11 meses-, Nivel Medio Menor — desde 2 años hasta 2 años y 11 meses - y Nivel Medio Mayor - desde 3 años hasta 3 años y 11 meses. La política educativa en estos cursos garantiza la gratuidad en la ROP, seleccionando en la admisión a las familias desaventajadas por criterios de vulnerabilidad. Esto permite que familias desaventajadas, por discriminación positiva en el acceso, no tengan que financiar con sus recursos la educación y alimentación de sus niños (Mateo \& Vásquez, 2016; Staab, 2017). Así, se traspasa el costo familiar a un financiamiento a la demanda por asistencia o basal dependiendo de la provisión (Ministerio de Educación et al., 2005).

La provisión de la ROP se compone de dos instituciones: la Junta Nacional de Jardines Infantiles (en adelante, Junji) y la fundación Integra. A su vez, estas instituciones pueden administrar o subvencionar centros ${ }^{1}$.

1 Los centros subvencionados por la Junji son denominados Vía Transferencia de Fondos (VTF) y los centros subvencionados por Integra son denominados Centros de Administración Delegada (CAD). 
Los centros administrados por la Junji o Integra tienen un financiamiento basal, es decir, un monto fijo mensual, mientras que los centros subvencionados por la Junji o Integra reciben un voucher por asistencia mensual promedio. El crecimiento de provisión ha seguido la estrategia de la privatización y descentralización implementada en toda la educación chilena (Bellei, 2007; Brunner et al., 2006; Corvalán et al., 2016; Seppänen et al., 2015). Se han aumentado los centros subvencionados de un $9 \%$ a un $46 \%$ entre los años 2001 y 2016 (Ministerio de Educación, 2002; 2017), siendo la provisión de la ROP fundamentalmente privada, subvencionada y heterogénea (Adlerstein \& Pardo, 2019; Cunill et al., 2017).

Los montos de la subvención varían según institución (la Junji o Integra) y cursos. La subvención es entregada en su totalidad si el porcentaje de asistencia promedio mensual corresponde al $75 \%$ de los niños matriculados. Esto significa que, a menor asistencia de los niños, menor es el porcentaje de subvención recibida por los centros. Para no perjudicar la subvención en los meses de invierno, las instituciones promedian la asistencia de los tres últimos meses para la asignación de recursos (Junta Nacional de Jardines Infantiles, 2016). El marco legal y normativo de las transferencias y subvenciones implementadas en la Junji e Integra es modificado anualmente según el presupuesto nacional y normativas institucionales; para profundizar en este aspecto se pueden consultar los manuales de la Junji (2019) e Integra (2019).

A pesar de la importancia de la asistencia en la subvención de los centros en la ROP, existen pocas investigaciones empíricas sobre su comportamiento. En términos generales, se ha identificado que existen retiros $u$ abandono de las familias relacionados principalmente con el cambio de domicilios y enfermedades de los niños (Castillo et al., 2007; Junta Nacional de Jardines Infantiles, 2010; 2017). Además, estos estudios no permiten identificar diferencias entre los sistemas basales o de voucher en el financiamiento de la ECE en la ROP (Cunill et al., 2017). No obstante la poca evidencia, existen propuestas para implementar el voucher en toda la ROP (Tokman, 2010) o combinar, para los centros subvencionados, un financiamiento basal fijo con una variabilidad por asistencia (Treviño, 2017).

Este artículo permite avanzar en resultados empíricos al examinar la institucionalidad de la ECE en Chile, identificando mecanismos causales que permiten explicar prácticas desde las distintas subjetividades: administradores, equipos pedagógicos y familia. Es decir, permite una visión sistémica que incluya tanto políticas de financiamiento, implementaciones en los centros, prácticas cotidianas y experiencias de las familias (Kagan et al., 2016). Finalmente, estos resultados nos permitirán identificar cómo se promueve el derecho a la educación y la ciudadanía en la primera infancia. 


\section{Metodología}

Utilizando la teoría del realismo crítico (Archer et al., 1998; 2016; Bhaskar, 1975/2008) se generan mecanismos causales a partir de estrategias y análisis propios de las investigaciones cualitativas, los que permiten contextualizar y conceptualizar el fenómeno estudiado (Barco \& Carrasco, 2018; Maxwell, 2004; Parra, 2016; Scott, 2014).

Para este artículo se consideran tres estrategias de contextualización: una observación intensiva (Scott, 2014), con visitas de uno a dos días por semana a cinco centros subvencionados en la localidad, entre octubre del 2015 y octubre del 2016; una producción de datos ricos o descripción densa (Geertz, 1973), con observaciones directas y no participantes (Creswell, 2007; Stake, 1998) a un centro un día a la semana, toda la jornada laboral, durante cinco meses; $y$, narrativas de conexión con entrevistas semiestructuradas (Flick, 2009) a administradoras, directivos, equipo pedagógico y madres de los cinco centros subvencionados $(N=40)$. Estas estrategias son una parte de las utilizadas en la tesis doctoral de la autora, a partir de una aproximación etnográfica llevada a cabo durante octubre de 2015 y octubre de 2016 en una localidad al sur-poniente de la Región Metropolitana en Chile. Todos los participantes firmaron un consentimiento sobre los objetivos del estudio, divulgación de los datos en publicaciones científicas y anonimato. Una limitante del estudio fue no incluir a familias que, estando matriculadas en los centros, dejan de ir, es decir, "niños y niñas desertores" en los centros subvencionados, debido a la alta movilidad de las familias en la localidad estudiada.

Posteriormente, se realizaron tres análisis de proceso para conceptualizar los resultados. Primero, la identificación de semirregularidades, con patrones o tendencias de eventos investigados, y que por sí sola muestra un alcance restringido, parcial e inestable (Lawson, 2003). Las semirregularidades en análisis cuantitativos pueden aparecer como tendencias estadísticas, mientras que en los análisis cualitativos son consideradas como temas (Fletcher, 2020). Luego, la abducción, que corresponde a la interpretación de los eventos sin recurrir a las teorías o conceptos preexistentes sobre el fenómeno (Blaikie, 2007; Danermark et al., 2002). Y, finalmente, la retroducción, que identifica las condiciones contextuales necesarias para entender el evento (Fletcher, 2017; Parra et al., 2020).

La identificación de semirregularidades buscó detallar en cada centro regularidades con respecto al financiamiento y, luego, compararlas entre ellas. Para concretar este paso, se utilizó el software Nvivo para identificar contenidos emergentes, temas y contenidos generales. Con la identificación de la asistencia como una variable clave, se realizó un análisis exploratorio 
descriptivo e histórico en un centro. A partir de los datos cualitativos y cuantitativos, se identificaron prácticas dentro de los centros subvencionados. Debido a los pocos resultados empíricos de investigaciones anteriores se utilizaron estrategias creativas (abducción) en conjunto con la identificación del contexto que posibilitan o no (retroducción) estas explicaciones.

\section{Resultados}

Estos resultados presentan las subjetividades de administradores, directivos, equipo educativo y familias en relación con la institucionalidad del financiamiento de la ECE, en especial en los centros subvencionados para las familias desaventajadas en Chile. A partir de ellos, podremos identificar cómo se promueve el derecho a la educación y la ciudadanía en la primera infancia.

\section{Análisis de la asistencia}

Para analizar la asistencia en los centros subvencionados se realizó un estudio exploratorio descriptivo con la información histórica de un centro a nivel general, por curso y mensual, desde marzo del año 2012 —año de inicio del funcionamiento - hasta noviembre del año 2015. El porcentaje de asistencia se calculó según el instructivo para otorgar la subvención: (total de asistencia de los niños / número de niños matriculados) / días trabajados. No se consideraron los días de vacaciones o de suspensión de clases, según el instructivo.

Los resultados de este análisis muestran un aumento del porcentaje de asistencia general del centro de un 66\% en el año 2012 a un $72 \%$ en el año 2015. Sin embargo, esta asistencia es diferente en cada curso y durante el año. Sala Cuna Menor presentó una disminución en los porcentajes de un $67 \%$ en 2012 a un 62 \% en 2015. Sala Cuna Mayor y Nivel Medio Menor aumentaron sus porcentajes de asistencia en los tres primeros años desde un $64 \%$ en el 2012 hasta un $75 \%$ en el 2014, para luego disminuir en el año 2015 a un 73\%. La situación contraria se presentó en Nivel Medio Mayor, que aumentó en todo el período su asistencia anual, desde un $68 \%$ en 2012 hasta un 78\% en 2015. En relación con los meses, en todos los cursos se observó una brusca disminución en los meses de invierno, y en especial en julio. Tanto para Sala Cuna Menor — con el más bajo promedio de asistencia anual - como para el curso de Nivel Medio Mayor — con el más alto promedio de asistencia anual—- en los meses de julio en todo el período analizado, la asistencia llegó en ambos cursos al $40 \%$ promedio.

Estos resultados empíricos e históricos muestran que la asistencia tiene un comportamiento móvil, que se diferencia por cursos y por períodos en el año, disminuyendo en todos los cursos a un $40 \%$ en los meses de 
julio. Las variaciones anuales en la asistencia crean ciertas prácticas desde los centros hacia las familias, y desde las administraciones hacia los centros subvencionados.

\section{Presión económica hacia los centros}

Las administraciones de los centros subvencionados han incorporado bonificaciones económicas para el equipo educativo, relacionadas con la asistencia. Dentro del Proyecto Educativo Institucional (PEI), que debe informar cada centro a su administración anualmente, se encuentran incorporados indicadores asociados al cumplimiento del $75 \%$ de la asistencia promedio por centro y por curso:

Cada objetivo del PEl tiene una nota con un porcentaje, este porcentaje más la asistencia personal de cada educadora y una evaluación personal, conforman un bono de desempeño que equivale como máximo al $80 \%$ del sueldo. El bono es personalizado según la asistencia de cada educadora y evaluación, pero los objetivos son iguales para todas (directora, C1).

Además, la administración entrega informes de gastos anuales, detaIlando las diferencias que deben asumir cuando no es completada la subvención. Esto remarca la importancia de la asistencia en la subvención para el funcionamiento del centro. El informe detalla que la mayoría de los gastos son financiados por la subvención, mientras que el resto lo asume la administración y otras iniciativas:

La subvención Junji es de un $55 \%$ y lo otro tiene que ver con donaciones, programas de recaudación y la red social. Entonces, el $45 \%$ lo pone la administración y el $55 \%$ lo pone Junji (directora, C1).

Si uno logra el $75 \%$ de asistencia, paga el $100 \%$ de las subvenciones, pero si tiene un $74,8 \%$, paga por niño asistido, y para efectos monetarios, no es rentable, para efectos empresa, si lo vemos así en números, en tema dinero no es rentable porque me pagan por quince niños y no los veintiocho... Entonces, para efectos de sueldo y todo lo que tienen que pagar no conviene (directora, C4).

Las administraciones traspasan las variaciones de las asistencias a objetivos económicos. Por un lado, identifican los montos asociados al funcionamiento del centro y las diferencias que existen con respecto a la subvención, mientras que, por otro lado, presionan al equipo con bonificaciones personales. Esto crea una presión desde las administraciones hacia el equipo pedagógico por mantener las asistencias sobre el $75 \%$. 


\section{Presiones punitivas hacia la familia}

Un hito clave al comienzo del año académico corresponde a la primera reunión con las familias. Esta reunión tiene como objetivo dar a conocer deberes y normativas del centro, entre ellas la cantidad de inasistencias injustificadas:

Reunión inicio de año a todas las familias. Uno de los deberes que deben asumir las familias es tener una asistencia constante. Eso significa no faltar más de 4 veces en el mes para Niveles Medios y 5 veces al mes en Salas Cunas. Al faltar más de esos días sin justificación, se procederá a condicionar y luego cancelar la matrícula. La directora dice: "Aprovechen el cupo en la Salas Cunas, porque hay una gran lista de espera" (observación, C1).

Las reiteradas inasistencias sin justificar autorizan a la directora a cancelar la matrícula y otorgar este cupo a otra familia en lista de espera. La cancelación de la matrícula no es inmediata. La familia es informada por carta y luego la directora realiza una visita domiciliaria para conocer el entorno donde habita el niño. A partir de estas acciones, se pueden generar nuevos compromisos con las familias que permitan regular la asistencia al centro:

Conversación directora y asistente social. Hablan de Jeremy. Su comportamiento es agresivo. Tiene padres drogadictos y viven con la abuela materna. Se preocupan por sus constantes faltas de asistencia. Es necesario enviar una carta: si sigue faltando se le cancelará la matrícula. Ambas se ponen de acuerdo en realizar una visita a la familia el día jueves, la idea de ambas es ir a ver en las condiciones que viven: salud de la madre, consumo de cigarros y condición habitacional. Se acuerdan de un caso parecido el año pasado, en donde la condicionalidad de la matrícula permitió mejorar la asistencia (observación, C1).

La advertencia de la cancelación de matrícula y las visitas domiciliarias son prácticas que buscan corregir las inasistencias injustificadas. Estas prácticas son consideradas como intromisión en la vida privada, que podrían generar continuas visitas de seguimiento:

Yo no iba a mandar a Emilia al centro, porque yo no trabajo, entonces no tenía necesidad de mandarla, pero la Educadora de Párvulos me dijo que si yo no la mandaba me iba a hacer un seguimiento con la asistente social y me iba a ir a visitar (madre 29, C4).

La cancelación de la matrícula y las visitas domiciliarias crean una presión de los centros hacia las familias por mantener una asistencia. Estas medidas 
son de carácter punitivo, al existir una consecuencia si no asisten regularmente al centro. Por el contrario, existen otras prácticas de los centros que se centran en flexibilizar las normativas que son consideradas como un apoyo por las familias.

\section{Flexibilizar para apoyar a las familias}

Entre las prácticas dirigidas a incentivar la continuidad de las familias, se encuentran la flexibilización del horario de ingreso o retiro de los niños, la posibilidad de participar solo en el almuerzo y presentar licencias médicas para no asistir:

La licencia médica es para justificar los días que no asisten. Porque acá los papás tienen claro que si ellos tienen más de tres días faltando, el niño se deserta y se hace correr la lista de espera. Obviamente, nosotros somos más flexibles, pero les decimos eso para que se asusten y quieran que les guardemos el cupo (directora, C3).

Y hay otras mamás que vienen a almorzar, porque ejemplo cuando están con licencia o están enfermitos avisan si lo pueden traer a almorzar y ellos llegan como a las doce (directora, C5).

Las familias consideran estas prácticas como un apoyo para cumplir con la asistencia, y la flexibilización de las normativas permite la continuidad sin recurrir al retiro o cancelación de la matrícula:

El centro tiene la opción de entrar más tarde si está muy helado, o que la pediatra le dé una licencia por todo el invierno y después vuelva otra vez en septiembre... Le guardan el cupo, o puede venir medio día, o como a las once y sale a las cuatro y media, que no está tan helado... Ahí le acomodan el horario por ser crónico, así no se enfermaría tanto, y no tendría que salir tan temprano (madre 9, C2).

Me dijeron que la única manera que la pudieran sacar es si ella falta, por ejemplo, una semana, o dos semanas o un mes y yo no avisara el por qué, me podrían sacar el cupo. Pero si yo les digo, por ejemplo, la voy a llevar a almorzar estos días porque está enferma y aquí tengo la licencia, no se toma en cuenta y mantiene el cupo (madre 22, C4).

Estas prácticas de flexibilización de las normativas y apoyo a la asistencia de las familias se realizan principalmente en los meses de invierno, cuando la variación de las asistencias aumenta en todos los cursos. Cambia 
la acción punitiva por una de apoyo que permita la continuidad de los niños en el proceso pedagógico.

\section{Discusiones}

Los resultados reportados en este artículo aportan nueva evidencia sobre la institucionalidad del financiamiento en los centros subvencionados para familias desaventajadas en la ROP de Chile y las subjetividades de administrativos, directivos, equipo educativo y familias. El análisis exploratorio descriptivo histórico nos entrega evidencia de que existen variaciones anuales y que en los meses de julio se registra en promedio un $40 \%$ de asistencia en todos los cursos. Es decir, el retiro o las inasistencias por cambio de domicilio y enfermedades de los niños identificados en otras investigaciones (Castillo et al., 2007; Junta Nacional de Jardines Infantiles, 2010; 2017) no son eventos aislados, por lo que es necesario incluirlos dentro de los análisis de las políticas educativas en la ECE.

El sistema de financiamiento de los centros, subvencionado mediante el voucher por asistencia o subvención a la demanda, presiona a las familias, centro y administración para mantener la asistencia. El centro debe rendir cuentas sobre la asistencia de los niños, que al ser menor del $75 \%$ afectará el funcionamiento y la bonificación personal. El equipo educativo, a su vez, presiona a las familias, limitando el número de días en que pueden faltar injustificadamente con cancelación de matrícula o visitas domiciliarias. Pero también flexibiliza las normativas en los períodos del año en los que la asistencia se vuelve crítica, lo cual es un apoyo para las familias, permitiendo un horario de ingreso o retiro de los niños personalizado, la posibilidad de participar solo en el almuerzo y de presentar licencias médicas para no asistir sin cancelar la matrícula.

Si bien la flexibilización de las normativas no permite la implementación total de las planificaciones, sí mantiene un vínculo de la familia con el centro y, con ello, la posibilidad de implementar las rutinas o actividades en el hogar, conservando el objetivo pedagógico. Es decir, dentro de las prácticas analizadas aquellas que apoyan la continuidad de las familias permiten mantener el derecho a la educación y, con ello, la continuidad en el acceso en períodos de baja asistencia. Sin embargo, la ciudadanía de los niños y niñas de 0 a 3 años de edad se ve condicionada por prácticas de interdependencia generacional — familia, centro, administradores - y de contexto — financiamiento, políticas educativas-, ejerciendo su poder asimétrico sobre la primera infancia (Devine, 2002). 


\section{Conclusiones}

A partir de los resultados sobre la institucionalidad del financiamiento en los centros subvencionados para familias desaventajadas en la ROP de Chile y las subjetividades de administrativos, directivos, equipo educativo y familias, se identifican dos mecanismos causales: variabilidad de la asistencia y lógica de control económico.

Gracias a la identificación de semirregularidades en los análisis de datos cuantitativos y cualitativos se pudo identificar la importancia de la asistencia en los centros subvencionados. Si bien otras investigaciones identificaban retiros, inasistencias por cambio de domicilio o enfermedades (Castillo et al., 2007; Junta Nacional de Jardines Infantiles, 2010; 2017), no eran considerados como una regularidad. La variabilidad de la asistencia es parte del contexto de las familias desaventajadas (retroducción), debido a la inestabilidad laboral, de vivienda y relaciones familiares. Se pudo comprobar que no representaban un hecho aislado y que debía incluirse en los análisis de la primera infancia y la ECE (abducción).

Los resultados sobre prácticas se identificaron a partir de los datos cualitativos con semirregularidades. Luego se identificó que la institucionalidad en el financiamiento, es decir, el contexto y normativas que rigen a los centros subvencionados (retroducción), fomentaba prácticas con presiones para mantener la asistencia. Pero que también existen prácticas de apoyo a las familias que flexibilizan las normativas en tiempos de baja asistencia (abducción). Es decir, la institucionalidad del financiamiento en los centros subvencionados instala una lógica de control económico, que genera prácticas, de presión o apoyo, orientadas a mantener la asistencia.

Ambos mecanismos causales son contradictorios. El financiamiento a los centros subvencionados no contempla la variabilidad de la asistencia, imponiendo una lógica de control económico, sin considerar el contexto de las familias desaventajadas en el que se desarrolla la ROP en Chile. Estos resultados permiten incluir a la institucionalidad del financiamiento como barrera de acceso dentro de los análisis de costo o asequibilidad.

Los resultados nos muestran cómo el derecho a la educación y la ciudadanía en la primera infancia de Chile se sitúan dentro de una institucionalidad de financiamiento que, si bien no considera costos a la familia, genera prácticas dirigidas a mantener la asistencia. Presiones y apoyos se encuentran relacionados con una "ciudadanía de consumo", en la que la asistencia es controlada y medida como producción en el derecho a la educación, condicionada por una interdependencia generacional. Por otro lado, las subjetividades de los agentes clave son normadas por la institucionalidad 
del financiamiento, identificando en la flexibilización de las normas la posibilidad de continuidad con el derecho a la educación y la ciudadanía.

Este artículo nos permite cuestionar el tipo de derecho a la educación y la ciudadanía que se encuentra promoviendo la institucionalidad del financiamiento en la primera infancia de Chile, por medio de las subjetividades de administrativos, directivos, equipo educativo y familias. A partir de ello, se propone avanzar en nuevas líneas de estudios sobre el derecho a la educación y la construcción de las ciudadanías en la primera infancia, sus interdependencias generacionales y contexto. Cuestiona también los límites de las normativas institucionales y cómo ellos pueden limitar o apoyar la construcción de ciudadanía en la primera infancia, además de los objetivos pedagógicos y curriculares. Estos resultados apuntan a erradicar el financiamiento por asistencia —o voucher-, ya que no promueve una justicia social en el acceso a familias desaventajadas, limitando el derecho a educación y ciudadanía.

\section{Sobre la autora}

Blanca Barco es doctora en Ciencias de la Educación de la Pontificia Universidad Católica de Chile, Chile. Master Recherche Sociologie Générale de la École des Hautes Études en Sciences Sociales (EHESS), Francia, y socióloga de la Universidad de Artes y Ciencias Sociales (ARCIS), Chile.

\section{Referencias}

Adlerstein, C. \& Pardo, M. (2019). La educación parvularia en Chile: del caleidoscopio de políticas a una institucionalidad sistémica. En A. Carrasco \& L. M. Flores (Eds.), De la reforma a la transformación: capacidades, innovaciones y regulación de la educación chilena (pp. 351-384). UC.

Archer, M., Bhaskar, R., Collier, A., Lawson, T. \& Norrie, A. (1998). Critical realism: Essential readings. Routledge.

Archer, M., Decoteau, C., Gorski, P., Little, D., Porpora, D., Rutzou, T. Smith, C., Steinmetz, G. \& Vandenberghe, F. (2016). What is critical realism? Perspectives: A Newsletter of the ASA Theory Section, Fall 2017. http://www. asatheory.org/current-newsletter-online/what-is-critical-realism

Ball, S. J. \& Vincent, C. (2005). The "childcare champion"? New labour, social justice and the childcare market. British Educational Research Journal, 31(5), 557-570. https://doi.org/10.1080/01411920500240700

Barco, B. \& Carrasco, A. (2018). Explicaciones causales en la investigación cualitativa: elección escolar en Chile. magis, Revista Internacional de investigación en Educación, 11(22), 113-124. https://doi.org/10.11144/Javeriana.m11-22.ecic

Bedregal, P. \& Pardo, M. (2004). Desarrollo infantil temprano y derechos del niño (Vol. 1). Serie Reflexiones: Infancia y Adolescencia n. ${ }^{\circ}$. Unicef. https:// www.unicef.org/chile/media/1171/file/desarrollo_infantil_temprano_y_dere chos_del_nino.pdf 
Bellei, C. (2007). Expansión de la educación privada y mejoramiento de la educación en Chile. Evaluación a partir de la evidencia. Revista Pensamiento Educativo, 40(1), 1-37. https://www.researchgate.net/publication/ 271831738_Expansion_de_la_educacion_privada_y_mejoramiento_de_la_ educacion_en_Chile_Evaluacion_a_partir_de_la_evidencia

Berlinski, S. \& Schady, N. (2015). Los primeros años: el bienestar infantil y el papel de las políticas públicas. BID.

Bhaskar, R. (1975/2008). A realist theory of science. Leeds Books.

Blaikie, N. (2007). Approaches to social enquiry: Advancing knowledge. Polity.

Blanco, M. R. (2005). La educación de calidad para todos empieza en la primera infancia. Revista Enfoques Educacionales, 7(1), 11-33. https://adnz.uchile. cl/index.php/REE/article/view/48175/50806

Brunner, J. J., Elacqua, G., González, S., Montoya, A. M. \& Salazar, F. (2006). Calidad de la educación. Claves para el debate. RIL.

Castillo, D., Santa Cruz, J. C. \& Yañez, N. (2007). Necesidades y expectativas de las familias atendidas por los jardines infantiles y salas cunas de la fundación Integra. Informe final. PIIE. http://archivospresidenciales.archivonacional. cl/uploads/r/archivo-presidencia-de-la-republica/1/f/7/1f7e18e35c 5120482789ce9b4f1ab8ba3d3dab9033a657ca0510b4825cf53810/DO_03_ Necesidades.expectativas.familias.pdf

Cockburn, T. (2013). Rethinking children's citizenship. Palgrave Macmillan.

Collombet, C. (2018). Les inégalités sociales d'accès aux modes d'accueil des jeunes enfants. Une comparaison européenne. Revue des Politiques Sociales et Familiales, 127(2), 71-82. https://www.persee.fr/doc/caf_2431-4501_2018_ num_127_1_3289

Corvalán, J., Carrasco, A. \& García-Huidobro, J. E. (2016). Mercado escolar y oportunidad educacional: libertad, diversidad y desigualdad. UC.

Creswell, J. W. (2007). Qualitative inquiry and research design: Choosing among five approaches. Sage.

Cunha, F., Heckman, J. J., Lochner, L. \& Masterov, D. V. (2006). Interpreting the evidence on life cycle skill formation. En E. A. Hanushek \& F. Welch (Eds.), Handbook of the economics of education (pp. 697-812). Elsevier. http:// jenni.uchicago.edu/papers/Cunha_Heckman_etal_2006_HEE_v1_ch12.pdf

Cunill, N., Leyton, C. \& Peralta, M. (2017). El peso de las asociaciones público privadas en los servicios para la primera infancia y los adultos mayores. Un estudio de casos en el marco de los derechos. Polis Revista Latinoamericana, 16(46), 239-269. https://dx.doi.org/10.4067/S0718-65682017000100239

Danermark, B., Ekström, M., Jakobsen, L. \& Karlsson, J. C. (2002). Explaining society. Critical realism in the social sciences. Routledge.

Del Boca, D. (2010). Child poverty and child well-being in the European Union: Policy overview and policy impact analysis-a case study: Italy. Tarki-Applica.

Delanty, G. (2003). Citizenship as a learning process: Disciplinary citizenship versus cultural citizenship. International Journal of Lifelong Education, 22(6), 597-605. https://doi.org/10.1080/0260137032000138158

Devine, D. (2002). Children's citizenship and the structuring of adult-child relations in the primary school. Childhood, 9(3), 303-320. https://doi.org/ $10.1177 / 0907568202009003044$ 
Devine, D. \& Cockburn, T. (2018). Theorizing children's social citizenship: New welfare states and inter-generational justice. Childhood, 25(2), 142-157. https://doi.org/10.1177/0907568218759787

Dominiciano, C. A. (2011). A educação infantil via programa bolsa creche: O caso do município paulista de Hortolândia. Educaçao em Revista, 27(3), 231-250. https://doi.org/10.1590/S0102-46982011000300012

Fletcher, A. (2017). Applying critical realism in qualitative research: Methodology meets method. International Journal of Social Research Methodology, 20(2), 181-194. https://doi.org/10.1080/13645579.2016.1144401

Fletcher, A. (2020). The reality of gender (ideology): Using abduction and retroduction in applied critical realist research. En M. van Ingen, S. Grohmann \& L. Gunnarsson (Eds.), Critical realism, feminism, and gender: A reader (pp. 205-224). Routledge.

Flick, U. (2009). An introduction to qualitative research. Sage.

Fondo de las Naciones Unidas para la Infancia (Unicef). (2007). Un enfoque de la educación para todos basado en los derechos humanos: un marco para la realización del derecho de los niños a la educación y los derechos dentro de la educación. Autor. https://www.observatoriodelainfancia.es/ficherosoia/ documentos/2410_d_Educacion_para_todos_UNICEF.pdf

Fundación Integra. (2019). Convenio de transferencia de recursos entre el Ministerio de Educación y la Fundación Educacional para el Desarrollo Integral de la Niñez, Integra. Autor. http://transparencia.integra.cl/transparencia_2015/ archivos/documentos/2019/Convenio\%20Transferencia\%20Integra\%20 2019.pdf

Geertz, C. (1973). The interpretation of cultures. Basic Books.

Ghysels, J. \& Van Lancker, W. (2011). The unequal benefits of activation: An analysis of the social distribution of family policy among families with young children. Journal of European Social Policy, 21(5), 472-485. https://doi. org/10.1177/0958928711418853

Guerrero, A. V. (2001). Educación y ciudadanía. Algunas disertaciones. Revista de Teoría y Didáctica de las Ciencias Sociales, (6), 87-101. https://www.redalyc. org/pdf/652/65200606.pdf

Heckman, J. J. (2006). Skill formation and the economics of investing in disadvantaged children. Science, 312(5782), 1900-1902. https://doi.org/10.1126/ science. 1128898

Heymann, J. (2003). Role of early childhood care and education in ensuring equal opportunity (Vol. 18). Unesco.

Junta Nacional de Jardines Infantiles (Junji). (2010). Comportamiento matrícula y asistencia niveles de educación parvularia Programas Educativos JUNJI. Autor.

Junta Nacional de Jardines Infantiles (Junji). (2016). Resolución Exenta 015/131. Aprueba texto refundido del Manual del Programa Transferencia de Fondos, desde la Junta Nacional de Jardines Infantiles a entidades sin fines de lucro, que creen, mantengan y/o administren Jardines Infantiles aprobados por la Resolución Exenta 015/159, de la Vicepresidenta Ejecutiva de la Junta Nacional de Jardines Infantiles, incorporando sus modificaciones posteriores. http://gobiernotransparente.junji.gob.cl/portal/transparencia/marco_nor mativo/2016/131.pdf 
Junta Nacional de Jardines Infantiles (Junji). (2017). Estudio sobre factores asociados a la permanencia de los párvulos en programas educativos de la Junji. Resumen ejecutivo. Autor.

Junta Nacional de Jardines Infantiles (Junji). (2019). Manual de transferencia de fondos. https://www.junji.gob.cl/wp-content/uploads/2020/09/619.pdf

Kagan, S. L., Araujo, M. C., Jaimovich, A. \& Aguayo, Y. C. (2016). Understanding systems theory and thinking: Early childhood education in Latin America and the Caribbean. En A. Farrell, S. L. Kagan \& E. K. M. Tisdall (Eds.), The Sage handbook of early childhood research (pp. 163-184). Sage.

Lawson, T. (2003). Reorienting economics. Routledge.

Lazzari, A. \& Vandenbroeck, M. (2012). Literature review of the participation of disadvantaged children and families in ECEC services in Europe. En J. Bennett (Ed.), Early childhood education and care (ECEC) for children from disadvantaged backgrounds: Findings from a European literature review and two case studies (pp. 27-47). European Commission. https://www. issa.nl/sites/default/files/pdf/Publications/equity/ECEC_for_childresn_case_ studies.pdf

Marshall, T. H. (1992). Citizenship and social class. Pluto.

Mateo, M. \& Vásquez, J. (2016). ¿Demanda insuficiente o insatisfecha? El caso de un programa municipal de provisión de guarderías en Chile. http://hdl. handle.net/10419/146495

Maxwell, J. A. (2004). Using qualitative methods for causal explanation. Field Methods, 16(3), 243-264. https://doi.org/10.1177/1525822x04266831

Meyers, M. K. \& Gornick, J. C. (2003). Public or private responsibility? Early childhood education and care, inequality, and the welfare state. Journal of Comparative Family Studies, 34(3), 379-411.

Ministerio de Desarrollo Social. (2018). Resultados Educación CASEN 2017. http:// observatorio.ministeriodesarrollosocial.gob.cl/storage/docs/casen/2017/ Resultados_educacion_casen_2017.pdf

Ministerio de Educación. (2002). Estadísticas de la educación año 2001. https:// centroestudios.mineduc.cl/wp-content/uploads/sites/100/2017/06/Estadisti casdelaEducacion2001.pdf

Ministerio de Educación. (2017). Estadísticas de la educación 2016. https://centroes tudios.mineduc.cl/wp-content/uploads/sites/100/2017/07/Anuario_2016.pdf

Ministerio de Educación, Junta Nacional de Jardines Infantiles \& Fundación Integra. (2005). Construyendo el futuro de los niños y niñas. Memoria 20002006. https://siteal.iiep.unesco.org/sites/default/files/sit_accion_files/siteal_ chile_0634.pdf

Moss, P. (2009). There are alternatives. Markets and democratic experimentalism in early childhood education and care (Vol. 53). Bernard Van Leer Foundation. https://files.eric.ed.gov/fulltext/ED522533.pdf

Organisation for Economic Co-operation and Development (OECD). (2001). Starting strong: Early childhood education and care. Autor. https://www.oecd. org/education/school/2535215.pdf

Organisation for Economic Co-operation and Development (OECD). (2006). Starting strong II: Early childhood education and care. Autor. http://www.oecd. org/education/school/startingstrongiiearlychildhoodeducationandcare.htm 
Organisation for Economic Co-operation and Development (OECD). (2017a). Education at a glance 2017: OECD indicators. Autor. https://read.oecd-ilibrary. org/education/education-at-a-glance-2017_eag-2017-en

Organisation for Economic Co-operation and Development (OECD). (2017b). Starting Strong 2017: Key OECD Indicators on Early Childhood Education and Care. Autor. https://www.oecd.org/education/school/starting-strong2017-9789264276116-en.htm

Parra, J. D. (2016). Realismo crítico: una alternativa en el análisis social. Sociedad y Economía, (31), 215-238. http://www.scielo.org.co/pdf/soec/n31/n31a10.pdf

Parra, J. D., Said-Hung, E. \& Montoya-Vargas, J. (2020). (Re)introducing critical realism as a paradigm to inform qualitative content analysis in causal educational research. International Journal of Qualitative Studies in Education, 34(2), 1-15. https://doi.org/10.1080/09518398.2020.1735555

Roose, R. \& De Bie, M. (2003). From participative research to participative practice: A study in youth care. Journal of Community \& Applied Social Psychology, 13(6), 475-485. https://doi.org/10.1002/casp.752

Scott, D. (2014). Ontology, epistemology, strategy and method in educational research. A critical realist approach. magis, Revista Internacional de Investigación en Educación, 7(14), 29. https://doi.org/10.11144/Javeriana.M7-14.OESM

Seppänen, P., Carrasco, A., Kalalahti, M., Rinne, R. \& Simola, H. (2015). Contrasting dynamics in education politics of extremes: School choice in Chile and Finland. Sense.

Staab, S. (2017). The expansion of childcare services (2006-2010). En S. Staab (Ed.), Gender and the politics of gradual change: Social policy reform and innovation in Chile (pp. 135-161). Palgrave Macmillan.

Stake, R. E. (1998). Investigación con estudio de casos. Morata.

Tokman, A. (2010). Compromisos y resultados en primera infancia: Progresos y obstáculos. Universidad Diego Portales.

Treviño, E. (2017). Financiamiento de la educación parvularia en Chile. Centro para la Transformación Educativa, Centre.

Van Lancker, W. (2013). Putting the child-centred investment strategy to the test: Evidence for the EU27. European Journal of Social Security, 15(1), 4-27. https://doi.org/10.1177/138826271301500103

Van Lancker, W. \& Ghysels, J. (2016). Explaining patterns of inequality in childcare service use across 31 developed economies: A welfare state perspective. International Journal of Comparative Sociology, 57(5), 310-337. https://doi. org/10.1177/0020715216674252

Vandenbroeck, M. \& Lazzari, A. (2014). Accessibility of early childhood education and care: A state of affairs. European Early Childhood Education Research Journal, 22(3), 327-335. https://doi.org/10.1080/1350293x.2014.912895

Wacquant, L. \& Bourdieu, P. (2005). Una invitación a la sociología reflexiva. Siglo XXI. 